\title{
Visual rating and volumetry of the medial temporal lobe on magnetic resonance imaging in dementia
}

The paper by Wahlund et al in this issue (pp 630-635) ${ }^{1}$ tries to shed light on the usefulness of biological markers in the routine diagnosis of Alzheimer's disease. Tissue loss in the medial temporal lobe regions as detected by MRI is one of the most feasible and accurate markers developed to date and investigating its usefulness in a clinical setting is potentially relevant.

The paper has two main merits. Firstly, it highlights that the usefulness of diagnostic examinations needs to be judged within the specific diagnostic pathway. ${ }^{2}$ For any given patient coming to medical attention, physical signs and instrumental examinations are aimed to increment the prior probability of one (or few) of various possible diagnoses until a threshold of probability is passed, and the patient is diagnosed as having a given disease. Obviously, those examinations most likely to enhance the diagnostic probability are privileged. In the case of the patient suspected of having Alzheimer's disease, a clinical assessment - including the mini mental state examination (MMSE) - is carried out first, usually followed by instrumental examinations, among which are CT or MRI. Wahlund et al show that, although low performance on the MMSE alone is able itself to detect a large proportion $(80 \%)$ of patients with Alzheimer's disease, the information on medial temporal lobe atrophy obtained from imaging leads to a significant increase of the detected proportion $(95 \%)$. The bottom line is that imaging has significant added diagnostic value and is therefore a clinically useful procedure.

The second merit is the comparison of the added diagnostic value of a complex technique with that of one that is more applicable to the clinical routine. The authors have measured medial temporal lobe atrophy both with computed volumetric analysis on digital MRI and visual rating on MR films. Whereas the first, a precious research tool, requires specific software and hardware machinery as well as trained personnel, the second relies solely on rater's expertise and conventional films. Importantly, the findings indicate that in a clinical setting the simpler technique is at least as accurate as the more complex.

Although the authors' attempt to investigate the relevance of common clinical procedures should be commended, some issues are open to further investigation. The separation of Alzheimer's from non-Alzheimer's dementias on the basis of the protocol of Wahlund et al was not satisfactory (accuracy of $71 \%$ ), suggesting that when it comes to separate different forms of dementia, atrophy in a single structure is not a good marker. Some findings indicate that the pattern of atrophy in some brain structures might be a more accurate differential marker. ${ }^{3}$ Secondly, the age of patients in the study of Wahlund et al was about 65 years, which is certainly not representative of most of the patients with Alzheimer's disease. Lastly, the value of imaging in addition to a complete neurogeriatric assessment ${ }^{4}$ was not considered by the authors, and more data are clearly needed on this issue.

GIOVANNI B FRISONI

Laboratory of Epidemiology and Neuroimaging,

IRCCS San Giovanni di Dio-FBF, via Pilastroni 1, 25123 Brescia, Italy

frisoni@master.cci.unibs.ithttp://www.centroalzheimer.org

1 Wahlund L-O, Julin P, Johansson S-E, et al. Visual rating and volumetry of the medial temporal lobe on magnetic resonance imaging in dementia: a comparative study. $\mathcal{F}$ Neurol Neurosurg Psychiatry 2000;69:630-5.

2 Hillman BJ. Medical imaging in the 21 st century. Lancet 1997;350:731-3.

3 Frisoni GB, Beltramello A, Geroldi C, et al. Brain atrophy in frontotemporal dementia. F Neurol Neurosurg Psychiatry 1996;61:157-65.

4 Chui H, Zhang Q. Evaluation of dementia: a systematic study of the usefulChui $\mathrm{H}$, Zhang Q. Evaluation of dementia: a systematic study of the useful-
ness of the American Academy of Neurology's practice parameters. Neurology 1997;49:925-35.

\section{Aneurysmal haemorrhage: partialling out the effect of the bleed}

The frequency and severity of persisting neuropsychological deficits after aneurysmal subarachnoid haemorrhage and associated surgical repair of the aneurysm are all too well known. ${ }^{1}$ What is less clear is the relative contribution of the initial bleed, widely assumed to be the most salient component in determination of cognitive outcome, versus factors associated with surgical and perioperative management. The Johns Hopkins group (this issue, pp 608-615) have evaluated cognitive outcome after surgical repair of ruptured $(n=27)$ and unruptured $(n=20)$ aneurysms in an attempt at partialling out the contribution of the subarachnoid haemorrhage from the appropriate neurosurgical intervention. ${ }^{2}$ Albeit in a relatively small series, patients with both ruptured and unruptured aneurysms performed, as a group, significantly below published norms on many neuropsychological tests postoperatively. Significant differences between preoperative and postoperative performance emerged in the unruptured aneurysm group on word fluency, verbal recall, and attentional/executive tasks. Performance of patients with ruptured aneurysms was in fact only significantly below that of patients with unruptured aneurysms on a few tests (verbal and visual memory).

The ISUIA study has reported that overall morbidity and mortality rates associated with surgical repair of unruptured intracranial aneurysms are higher than those reported previously. ${ }^{3}$ A considerable, albeit short term, negative impact on functional health and quality of life has also been identified after treatment of unruptured aneurysms. ${ }^{4}$ Prospective necropsy and angiographic studies indicate that between $3.6 \%$ and $6 \%$ of the population 
harbour an intracranial aneurysm. ${ }^{5}$ If the morbidity and mortality of elective surgical clipping of asymptomatic unruptured aneurysms are higher than we previously thought, then there are important implications for future clinical management and questions about the need (or not) for more accurate and complex non-invasive screening in concert with determination of parameters of risk.

As in surgical management of acoustic neuromas where hearing preservation has virtually replaced mortality, serious morbidity, and even facial nerve preservation, as a major outcome, enhanced surgical management of intracerebral aneurysms now requires more sensitive less physically based outcomes. The key challenge for applied neuropsychology over the next few years has to be to crystallise out clinically relevant cognitive measures which are comprehensible to our neurosurgical and neuroradiological colleagues and which allow more sensitive audit of interventions and refinement of current practice. In addition the roles of transcranial Doppler documented clinically significant vasospasm, nimodipine, hydrocephalus, and timing of surgery, still evade elucidation.

Interfacing with optimal surgical management of ruptured and unruptured aneurysms is the current revolution in Guglielmi coiling, which has been gathering momentum since the early 1990s. The International Subarachnoid Aneurysm Trial (ISAT) ${ }^{5}$ is a prospectively randomised controlled clinical trial funded by the United Kingdom Medical Resarch Council, the Canadian Medical Research Council, and the United Kingdom Stroke
Association. It aims to compare the safety and efficacy of endovascular treatment of ruptured intracranial aneurysms with conventional neurosurgical clipping in those cases where there is equipoise. A substudy of the main trial is evaluating cognitive outcome in both groups at 1 year follow up in 7 United Kingdom centres, aiming to recruit 150-200 patients in each group. As the largest consecutive series in the neuropsychological outcome of aneurysmal subarachnoid haemorrhage to date this may help with some of the unanswered questions. We may not be able to do anything about the initial bleed, but manipulation of surgical management (hypothermia, temporary clipping, timing of surgery) and maximising the potential of less invasive endovascular techniques are open to us.

KATHERINE CARPENTER

Russell Cairns Unit, The Radcliffe Infirmary, Oxford

katherine.carpenter@orh.anglox.nhs.uk

1 Ogden J, Utley T, Mee EW. Neurological and psychological outcome 4-6 years after subarachnoid haemorrhage. Neurology 1997;41:25-34.

2 Hillis AE, Anderson N, Sampath P, et al. Cognitive impairments after surgical repair of ruptured and unruptured aneurysms. F Neurol Neurosurg Psychiatry 2000;69:608-15.

3 The International Study of Unruptured Aneurysms Investigators. Unruptured intracranial aneurysms: risk of rupture and risk of neurosurgical tured intracranial aneurysms: risk of rupture

4 Raaymakers TWM, on behalf of the MARS Study Group. Functional outcome and quality of life after angiography and operation for unruptured come and quality of life after angiography and operation for unrupture
intracranial aneurysms. $₹$ Neurol Neurosurg Psychiatry 2000;68:352-353.

5 Wardlow JM, White PM. The detection and management of unruptured Wardlow JM, White PM. The detection and m
intracranial aneurysms. Brain 2000;123:205-21.

6 Molyneux AJ, Kerr RSC, Kerr MS. International Subarachnoid Aneurysm Trial. F Neurosurg 1999;91:352-3. 